\title{
Senolytic Development in Osteoarthritis: A New Paradigm
}

\author{
Christian Jorgensen ${ }^{1,2 *}$, MD, PHD \\ ${ }^{1}$ Inserm, U1183, Montpellier, F-34091, France \\ ${ }^{2}$ Université MONTPELLIER 1, UFR de Médecine, Montpellier, F-34000, France \\ *Corresponding author: Christian Jorgensen, France; Email: christian.jorgensen@inserm.fr
}

Received: December 24, 2021; Accepted: December 27, 2021; Published: December 31, 2021

\begin{abstract}
OA is a large unmet medical need, with increasing incidence in our aging population. Recent physiopathogical knowledge have identified the accumulation of senolytic cells in cartilage and the synovium as deleterious. This has prompted the development of senolytic drugs in OA. Senolytics are a class of drugs that selectively clear senescent cells. Senolytics induce apoptosis of senescent cells accumulated in joint tissue with a tissue-destructive SASP (senescent associated secreted proteome). They include three major classes of molecules. Senolytics which seek to kill/eliminate senescent cells by playing on increased cell survival pathways during the senescence process. Seno-morphics, which change the inherent properties of the senescent state, for example by reactivating autophagy while maintaining the stopping of the cycle. And finally, a third class of therapeutic agents, senosuppressants, will block the entry or the spread of senescence. Thus, drugs with senotherapy properties such as metformin, rapamycin, fisetin, dasatinib, quercetin, resveratrol, have shown modulation of the aging process and associated pathologies including OA. Thus, many therapeutic alternatives in the treatment of osteoarthritis are opening up, but clinical trials will have to be carried out to confirm their effectiveness.
\end{abstract}

Keywords: Senescence, Osteoarthritis, Senolytics

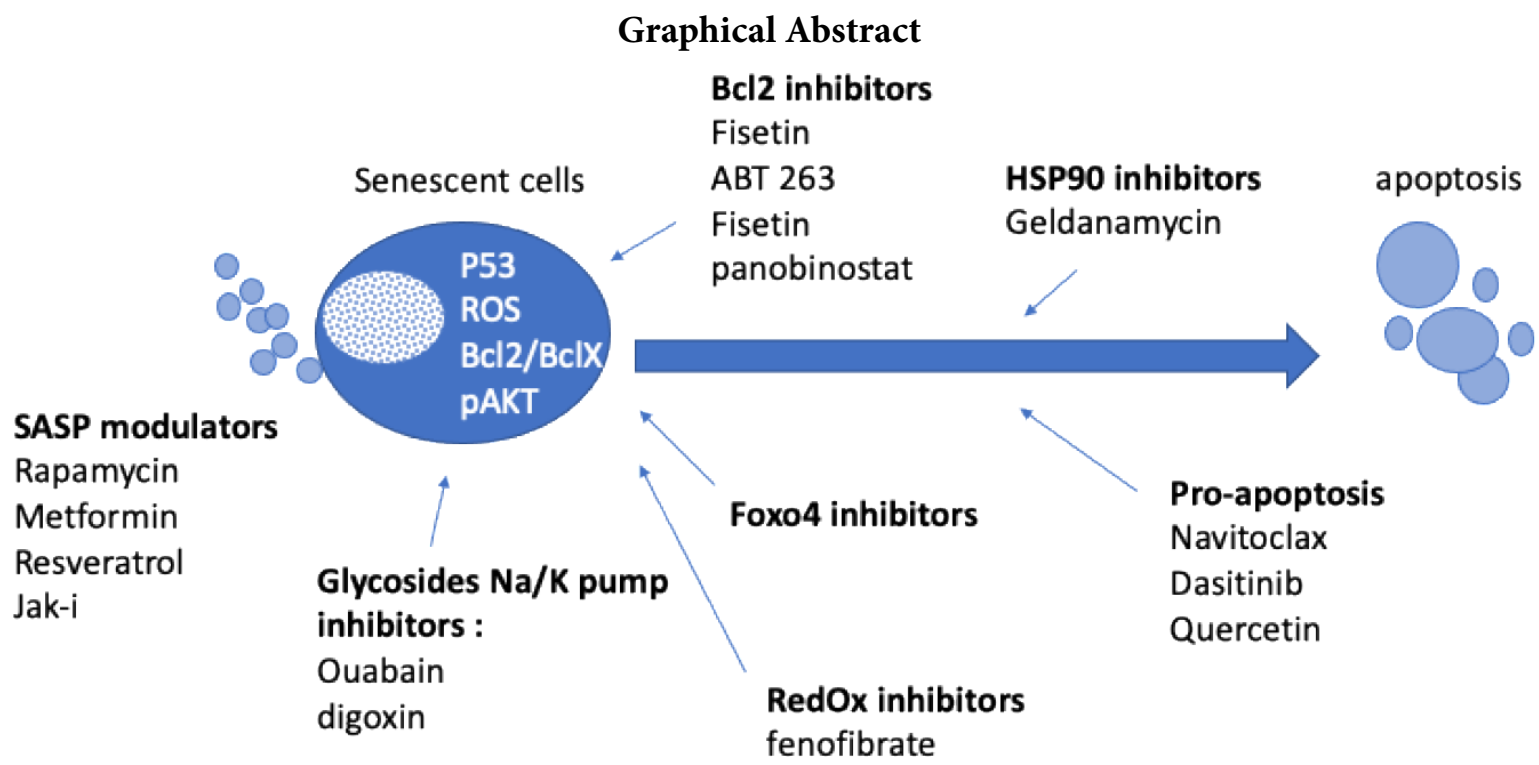

\section{Introduction}

Life expectancy of human populations is increasing worldwide as a result of medical and socio-economic advances, the years of life gained are often in poor health. Indeed, aging poses specific medical problems because it is the primary risk factor for developing a broad spectrum of chronic musculoskeletal diseases in the general population including osteoarthritis (OA) and osteoporosis [1]. Current

advances in the biology of aging will be at the origin of therapeutical improvement making it possible to increase life expectancy in good health and prevent $\mathrm{OA}$.

\section{Senescence and OA Physiopathology}

Senescence is a complex pathophysiological phenomenon that can be observed in the context of tissue damage associated with aging in the context of cancer development. Cellular senescence is a cell 
fate that entails essentially irreversible replicative arrest, sustained viability with resistance to apoptosis and increased metabolic and protein secretion activity [2].

Inducers of senescence can be DNA damage, telomere dysfunction, oncogene expression or replicative stress or even inducers of proliferation such as growth hormones. Extrinsic Inducers of senescence include the presence of circulating AGEs, saturated lipids and other bioactive lipids (prostaglandins), reactive metabolites (e.g. ROS, hypoxia), mechanical stress, inflammatory cytokines, damageassociated molecular patterns and pathogen-associated molecular patterns (PAMPs, e.g. bacterial endotoxins).

Cellular senescence results in cell cycle arrest by the activation of cyclin kinase inhibitor (including p16INK4a and p21Cdkn1a), associated with an increase in beta galactosidase activity and increased cell metabolism. This leads to the secretion of many secreted proteins that are calls SASP including proteins responsible for inflammation, insulin resistance, proteins that promote apoptosis, chemokines and factors of the TGF Beta family. The SASP profile varies depending on the cell type and will commonly include cytokines IL1, IL6, IL8, metalloproteases MMP13 secreted in the immediate cellular environment. Senescent cells accumulate with ageing and at causal sites of multiple chronic disorders, including musculoskeletal diseases associated with ageing, including $\mathrm{OA}$ and osteoporosis. The most deleterious senescent cells are resistant to apoptosis and have up-regulation of anti-apoptotic pathways allowing them to survive, despite killing neighbouring cells [3-5].

Osteoarthritis (OA) is a common pathology of cartilage, leading to disability and chronic pain. This incidence of OA increases with the aging of the population. It results on a complex physiopathology combining synovial inflammation, subchondral bone oedema and osteoblast activation and chondrocytes hypertrophy associated with the secretion of metalloproteases collagenases, ADAMT'S which will remodel cartilage extracellular matrix. This is accompanied by an arrest of the cell cycle, alteration of the cell metabolism as well as a chronic inflammation of the synovium. These biological events are related to the senescence of synovial cells and chondrocytes. The role of senescent cell accumulation in OA has been demonstrated in mouse models. Using elegant genetic constructs where senescent cells positive P16 very be selectively removed from articular tissues. In these experiments the elimination of senescent cells, in particular in joint tissues, helps prevent experimental osteoarthritis [6-8]. This suggest that drugs able to deleted senescent cells in osteoarthritis disease could provide clinical benefit.

\section{Senolytics in OA}

The demonstration of molecules capable of preventing senescence opens an important pathway in the treatment of diseases associated with aging. Senolytics induce apoptosis of senescent cells accumulated in joint tissue with a tissue-destructive SASP. Senolytics are a class of drugs that selectively clear senescent cells (SC) [9]. Through this demonstration of causality between senescence and tissue degeneration, a new category of treatments aimed at targeting senescence has recently emerged in preclinical and clinical studies.
They include three major classes of molecules. Senolytics which seek to kill/eliminate senescent cells by playing on increased cell survival pathways during the senescence process. Seno-morphics, which change the inherent properties of the senescent state, for example by reactivating autophagy while maintaining the stopping of the cycle. And finally, a third class of therapeutic agents, senosuppressants, will block the entry or the spread of senescence. Thus, drugs with senotherapy properties such as in particular metformin, rapamycin, fisetin, dasatinib/quercetin mixture, resveratrol, spermidine, curcumin and astaxanthin, have shown that the aging process and associated pathologies, can be partially prevented in mice. Moreover, 4 of these molecules are currently in phase I clinical trials in several indications in the elderly. Therefore, geroprotective interventions (senostatic strategies) are now designed to slow ageing and maintain health for longer before end of life.

The first studied senolytic drugs including Dasatinib, Quercetin, Fisetin are proapoptotic molecules. These drugs are MDM2 antagonists with ubiquitin ligase activity leading to the down expression of P53. These molecules therefore make it possible to switch senescent cells back to apoptotic cells and therefore eliminate accumulative senescent cells. Therapeutic strategies combining dasatinib and quercitin have been proposed with encouraging results, in particular in diabetes or dysmetabolic diseases $[10,11]$.

Other molecules have been identified as regulators of $\mathrm{Bcl} 2$ apoptosis pathway through $\mathrm{Bcl}$-xl inhibition. More recently, Nutlin3a (an MDM2 inhibitor) or navitoclax (a BCL-2 inhibitor), has been proposed as a alternative therapeutic strategy in osteoarthritis disease with encouraging preclinical results $[12,13]$. Navitoclax reduced the expression of inflammatory cytokines and promoted cartilage matrix aggregation in OA chondrocyte by inducing apoptosis. Moreover, OA pathological changes in the cartilage and subchondral bone in OA rat were alleviated by Navitoclax intra-articular injection. However phase 2 clinical study in OA was stopped prematurely because of lack of results.

Among other senolytics, rapamycin regulates cell metabolism (through the inhibition of mTor pathway) and induces autophagy [14]. Mildly lowering the activity of the nutrient-sensing network, especially the activity of mechanistic target of rapamycin protein complex 1 (mTORC1), using natural metabolites from the systemic environment that can rejuvenate stem cells, increasing autophagy/mitophagy, and reducing age-related inflammation are emerging as key mechanisms by which these interventions exert their effects. Rapamycin dramatically decelerated loss of proliferative potential caused by ectopic p21, p16 induction. During cell cycle arrest, rapamycin transformed the irreversible arrest into a reversible condition.

Other molecules, such as FOXO4-derived peptide designed to inhibit the interaction of this transcription factor with the tumour suppressor $\mathrm{p} 53$ reduced senescent cell viability through p53-mediated cell-intrinsic apoptosis [15]. In addition, FOXO4 inhibition in cells that were already senescent, but not their control counterparts, reduced viability and cell density. After acute damage FOXO4 favors senescence over apoptosis and maintains viability of senescent cells by repressing their apoptosis response. 
HSP90 inhibitors have been shown to be active in cancer through targeting of p53 and inducing apoptosis. Geldanamycin (GA), a benzoquinone ansamycin, depletes mutant $\mathrm{p} 53$ in breast cancer cell. GA restores ubiquitination and degradation of mutant $\mathrm{p} 53$ by the proteasome [16].

More recently, fibrates have shown senolytic activity. Fibrates impact on autophagy and lipid metabolism through PPAR ligands and constitution of retinoic acid heterodimer. Fibrate have a senolytic potential on OA senescent chondrocytes in-vitro. Peroxisome proliferator activated receptor alpha activation decreases synovial inflammation and cartilage lesions in osteoarthritis $[17,18]$.

Finally, seno-suppressants which directly inhibit SASP via inflammatory cytokine secretion related to the SASP may be proposed in degenerative joint diseases. Janus Kinases (JAK) are cytosolic protein tyrosine kinases directly associated with receptors for cytokines, hormones and growth factors involved in SASP. Jak inhibitors, such as Bariticinib or Ruxolitinib ${ }^{\circledR}$ reduce the expression of CKIs p16INK4a and p21Cdknla, correlating with the reestablishment of the proliferative capacity of senescent cells. Janus Kinase inhibitors have shown the ability to reduce adipocyte SASP and restore function and mobility in aged mice [19]. JAK1/2 inhibitors reduced inflammation and alleviated frailty in aged mice. Recently, Jak inhibitors was shown to reduce senescent cells in ageing tendon.

\section{Conclusion}

$\mathrm{OA}$ is a large unmet medical need. Recent physiopathogical knowledge have identified the accumulation of senolytic cells in cartilage and the synovium as deleterious. This has prompted the development of senolytic drugs in OA. Thus, many therapeutic alternatives in the treatment of osteoarthritis are opening up, but clinical trials will have to be carried out to confirm their effectiveness on a structural impact and not just anti-inflammatory (Figure 1).

\section{References}

1. Christensen K., Doblhammer G., Rau R (2009) Ageing populations: the challenges ahead. Lancet 374: 1196-1208. [crossref]

2. He S, Sharpless NE (2017) Senescence in health and disease. Cell 169: 1000-1011. [crossref]

3. Hernandez SA, Tristan VDJ, Simon M, Victor G, Judith C, et al. (2017) Unmasking Transcriptional Heterogeneity in Senescent Cells Curr Biol. 27: 2652-2660. [crossref]

4. Kirkland JL, Tchkonia T (2017) Cellular senescence: a translational perspective. EBioMedicine. 21: 21-28. [crossref]

5. Jeon OH, Kim C, Laberge RM (2017) Local clearance of senescent cells attenuates the development of post-traumatic osteoarthritis and creates a pro-regenerative environment. Nat Med 23: 775-781. [crossref]

6. Tachikart Y, Malaise O, Mumme M, (2019) Seno-suppressive molecules as new therapeutic perspectives in rheumatic diseases. Biochem Pharmacol 165: 126-133.

7. Jeon OH, David N, Campisi J, (2018) Senescent cells and osteoarthritis: a painful connection. J Clin Invest 128: 1229-1237. [crossref]

8. Patil P, Dong Q, Wang D (2019) Systemic clearance of p16 (INK4a) - positive senescent cells mitigates age-associated intervertebral disc degeneration. Aging Cell 18: e12927.

9. Kirkland JL, Tchkonia T (2020) Senolytic drugs: from discovery to translation. J Intern Med. 288: 518-536. [crossref]

10. Wu D, Prives C (2018) Relevance of the p53-MDM2 axis to aging. Cell Death Differ 25: 169-179. [crossref]
11. Wiley CD, Schaum N, Alimirah F (2018) Small-molecule MDM2 antagonists attenuate the senescence-associated secretory phenotype. Sci Rep 8: 2410-2414. [crossref]

12. Yosef R, Pilpel N, Tokarsky AR (2016) Directed elimination of senescent cells by inhibition of BCL-W and BCL-XL. Nat Commun 7: 11190-95. [crossref]

13. Hao Y, Cheng C, Hao C, Xiaojun D, Juan L (2020) Navitoclax (ABT263) reduces inflammation and promotes chondrogenic phenotype by clearing senescent osteoarthritic chondrocytes in osteoarthritis. Aging (Albany NY) 12: 12750-12770. [crossref]

14. Zoya ND, Svetlana GZ, Elena IB, Valery AP, Tatiana VP (2009) Rapamycin decelerates cellular senescence. Cell Cycle 12: 1888-1895

15. Baar MP, Brandt RM, Putavet DA (2017) Targeted apoptosis of senescent cells restores tissue homeostasis in response to chemotoxicity and aging. Cell 169: 132147. [crossref]

16. Fuhrmann SH, Ling YY, Zhao J (2017) Identification of HSP90 inhibitors as a novel class of senolytics. Nat Commun 8: 422-25. [crossref]

17. Clockaerts S, Bastiaansen JYM, Feijt C (2011) Peroxisome proliferator activated receptor alpha activation decreases inflammatory and destructive responses in osteoarthritic cartilage. Osteoarthritis Cartilage 19: 895-902. [crossref]

18. Nogueira RU, Lorenzo GI, Blanco FJ (2019) Fibrates as drugs with senolytic and autophagic activity for osteoarthritis therapy. EBioMedicine 45: 588-605. [crossref]

19. Chen M, Xiao L, Dai G, Lu P, Zhang Y, et al. (2021) Inhibition of JAK-STAT Signaling Pathway Alleviates Age-Related Phenotypes in Tendon Stem/Progenitor Cells Frontiers in Cell and Developmental Biology 9: 575-580 [crossref]

\section{Citation:}

Jorgensen C (2021) Senolytic Development in Osteoarthritis: A New Paradigm. Integr J Orthop Traumatol Volume 4(2): 1-3. 